\title{
THE RINGS OF URANUS
}

André BRAHIC, Observatoire de Paris, Université Paris VII

One of the very exciting discoveries in astronomy and planetary sciences in recent times is the detection of a series of narrow rings around Uranus. During more than three centuries, the rings of Saturn have had a special fascination and symbolism and an enormous amount of literature has been devoted to studies of their nature, properties and origin. The discovery of Uranus's rings, and two years later of Jupiter's rings has not only renewed interest but also raised a number of new cosmogonical questions.

Planetary rings are important not only because of the dynamical problems which they pose, but also because it is probable that processes which played a role in planet and satellite formation are still at work in these rings: rings systems are probably examples of arrested growth and they afford a good opportunity of studying some of the accretion mechanisms which operated in the early solar system. Planetary rings may also provide an appropriate analog for events in flat systems like spiral galaxies or accretion discs. Furthermore, particles of planetary rings are natural probes of the internal structure of the central planet.

Nobody knows if Uranian rings have been formed with Uranus $4.510^{9}$ years ago or are younger. Any model attempting to understand the origin of these rings must be considered as very uncertain. We do not discuss here the problem of the origin of planetary rings (break-up of a large body, condensation of the protoplanetary nebula, meteoroidal bombardment, or a combination of these processes). A number of problems like the interaction with interplanetary micrometeoroids (their amount is not well known at the distance of Uranus) or the interaction with Uranus magnetosphere (which is not known - Brown, 1976) are not considered here. In this book, Elliot 
has reviewed the data and analyses of observations. For earlier reviews, see Ip (1980), Dermott (1981b), and Brahic (1981). In this brief article, deductions from observations are first surveyed. Then, we confine ourselves mainly to a discussion of the dynamics of narrow rings and on ring-satellite interactions. Finally a brief comparison of Uranian rings with Jovian and Saturnian rings is reviewed and a number of unsolved problems and future observations are quoted.

It is interesting to note that Herschell thought in 1787 and 1789 that he saw two stubby rings around Uranus as shown in Figure 1.

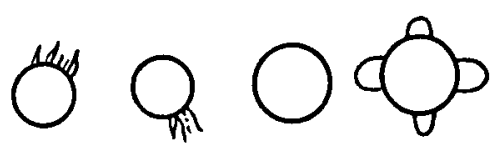

Figure 1. Herschell's drawings.

Further observations convinced him by 1798 that Uranus has no ring in the least resembling those of Saturn.

Many papers have been published just after the discovery of Uranian rings. I have no room here to quote all of them. Now, the dust has settled and I outline here only that which I consider as the most important results on ring dynamics, but I do not enter into any details of the models, which are described in the references at the end. I have only tried to emphasize the main physical arguments without any detailed calculation.

\section{DISCOVERY OF THE RINGS OF URANUS}

It is almost impossible to observe directly from the Earth a system of dark rings within 8 seconds of arc around a planet which has an apparent diameter of 4 seconds of arc. The Uranian rings have been discovered during the occultation by Uranus of the late type star SAO 158687 of visual magnitude -9.5 on March 10,1977 . Highspeed photoelectric photometry of occultations provides a powerful tool for probing the upper atmosphere of a planet, as has been shown during the past decade from occultations involving Mars, Jupiter, and Neptune. When the occultation of a reasonably bright star by Uranus was predicted, teams of observers from United States, France, India, China, Japan, Australia, South Africa planned to travel to Africa, South Asia, and Australia, where the event would be visible. In addition, arrangements were made to fly N.A.S.A. Kuiper Airborne 
Observatory with its $91-\mathrm{cm}$ telescope to the southern hemisphere. All of these efforts paid off when not only the predicted occultation by the planet, but also a series of secondary occultations by the previously unsuspected rings, were observed. The more complete observations have been made at that time by Elliot and his team observing from the N.A.S.A. plane over the southern Indian Ocean. It is interesting to note that the recorded conversation (Elliot et al., 1977a) among the observers shows that they progressively changed from skepticism to unbounded enthusiasm. Since, seven useful occultations have been observed and the structure of the rings is now known with an accuracy of the order of a few kilometers and less. It is pleasant to note that occultations techniques are very powerful: they give a resolution slightly better than the one obtained from a Voyager type spacecraft flying-by the planet. From the Earth, the resolution is limited by Fresnel diffraction and by the angular size of occulted star at the distance of Uranus. The "best" stars tend to be giant stars, giving us a resolution limit of a few kilometers. A more detailed review of the observations is given in Elliot's paper in this volume. Here I concentrate only on the main deductions from observations.

\section{DEDUCTIONS FROM OBSERVATIONS}

At least nine narrow rings encircle the planet, extending between 1.60 and 1.95 planetary radii and identified in order of increasing planetary distance $(6,5,4, \alpha, \beta, \eta, \gamma, \delta, \varepsilon)$. Compared to their circumference (some $250000 \mathrm{~km}$ ), they are exceedingly narrow: most do not exceed 10 kilometers in width and only one, the outermost ring, spans as much as $100 \mathrm{~km}$. Three rings are circular, but an unexpected revelation is that at least six have eccentricities of 0.001 to 0.01 and have variable widths. Both of these characteristics are best illustrated by the external ring, called $\varepsilon$. It is the largest and the best known: its distance from Uranus varies by about $800 \mathrm{~km}$ and its width changes from 20 to $100 \mathrm{~km}$ linearly with its distance from Uranus. This structure can be simply explained if ring boundaries are, as shown in Figure 3, Keplerian ellipses with same apsidal line, the eccentricity of the outer ellipse being a little 


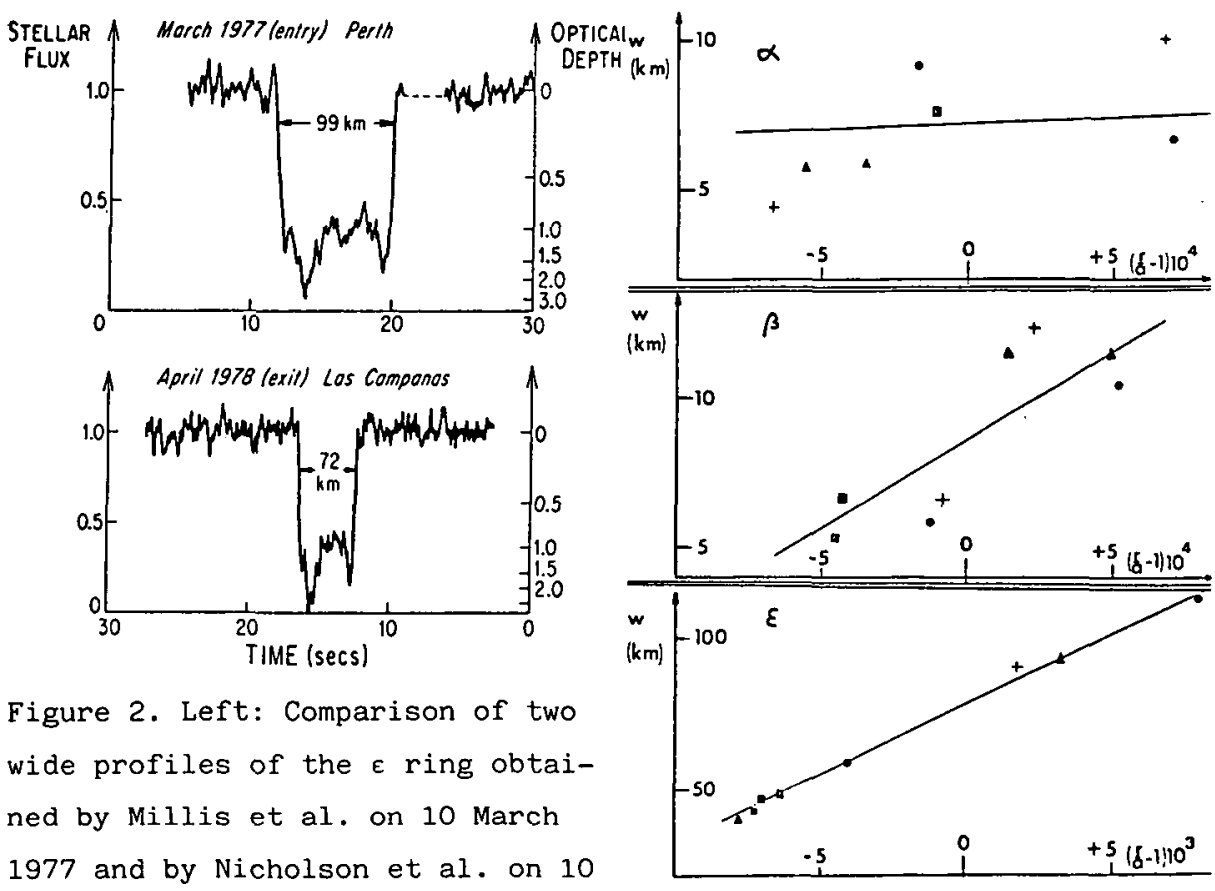

April 1978. (After Nicholson et al., 1978). Right: Radial width of the rings $\alpha, B$ and $\varepsilon$ plotted against radius for 7 occultations. (After Sicardy et al., 1981).

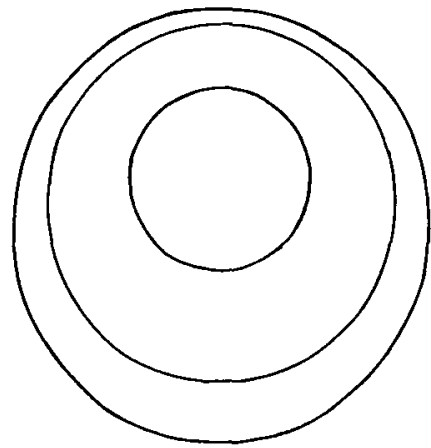

Figure 3 . Shape of $\varepsilon$ ring.

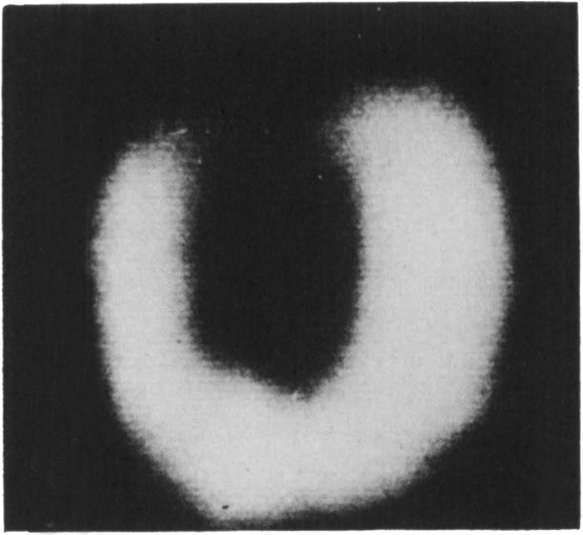

Figure 4. First picture of Uranus rings. (Nicholson et al., 1978).

bigger than the one of the inner ellipse.

The remarkable thing is that these elliptic rings precess slowly about the planet, just as they should due to the planet's oblateness. For example, the $\varepsilon$ ring precesses at a rate of $1.364^{\circ}$ 
per day. Normally, the rate of precession would depend on the distance to the planet: the inner edge of a ring of any width would precess more rapidly than its outer boundary. This differential precession would shear each ring into a circular band in a time scale of only a few tens of years. In fact, each ring precesses as a rigid whole, the eccentric nature of the rings seems to be continually reinforced, either by satellites or by the ring material itself. The profile of the rings looks the same everywhere (Figure 2). The rings have sharp outer edges and structure bigger than noise appears within a number of rings.

The rings are really dark: they reflect less than a few percent of the light that strikes them: their visual albedo is of the order of 0.025 . This low albedo implies that the majority of ring particles lack coatings of water, ammonia or methane frosts, materials which are common among many of the outer-planet satellites, including the Uranian satellites.

Near 2.2 microns, the methane absorption is enormous. It seems there are almost no clouds in the atmosphere of Uranus and either ammonia frosts or certain hydrated silicate minerals could cause these well-defined absorption features. At 2.2 microns, the albedo of Uranus is only $10^{-4}$ and the rings are brighter than the planet. Occultations must be observed at this wavelength.

Because of their proximity to Uranus, small cross-section, and low albedo, the rings are extremely difficult to see. In fact, using the 5-meters telescope at Palomar mountain, Matthews, Nicholson and Neugebauer (1981) have obtained in 1978 the first picture of the ring system by substracting sets of scans made at 1.6 microns (where the planet is brighter than the rings) from corresponding scans at 2.2 microns (where the planet absorbs virtually all the light that strikes it). The $\varepsilon$ ring has the most important contribution to this image. Its signature can be seen clearly: the bottom of the image corresponds to its wide part and the top to the narrow part (Figure $4)$. It is not yet understood why the image is slightly brighter on the right. It seems that there is less material between the rings than in the rings. An evensmaller amount of material could easily dominate if it was there. The fact that we see the signature of the 
rings indicates that spaces between the rings are relatively free of debris.

If we plot the precession of the $\alpha, \beta$ and $\varepsilon$ rings against semimajor axis, we get a result just as it should be if it was a precession around an oblate planet. Any satellite able to affect this precession rate should be seen from the Earth. A value of 0.0034 for $\mathrm{J}_{2}$ (Nicholson et al. 1978). From $\mathrm{J}_{2}$ and the optical oblateness (difficult to observe from the Earth), the period of rotation of the planet can be deduced if hydrostatic equilibrium holds. A rotation period of $15.5 \mathrm{~h}$ has been obtained by Elliot et al. (1981). Elliot et al (1981) give a value of $(-2.9 \pm 1.3) \cdot 10^{-5}$ (see also Nicholson et al., 1981) for $\mathrm{J}_{4}$. This value is consistent with several interior models. If the error bar could be reduced by an order of magnitude, it could become an useful discriminator for interior models.

Additional features like detailed structure inside some rings (similar to the Saturn F ring?) and broad components outside two or three rings (Elliot et al,,1981 and Sicardy et al,,1981) have also to be explained. Figure 5. A broad component has been observed at the. outer edge of $n$ ring. Others are suspected close to 6 and $\delta$ rings. (After Sicardy et al., 1981).

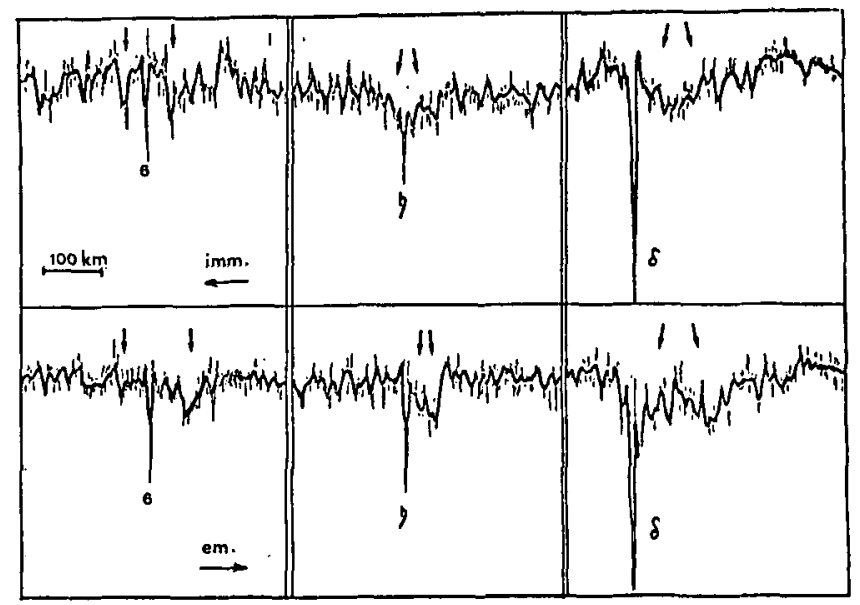

Details on the data of the observed occultations can be found in the litierature (Bhattacharyya \& Bappu; 1977; Chen et al., 1978 Elliot et al., 1977,1978,1980,1981'; Hubbard \& Zellner, 1980; Mahra \& Gupta, 1977; Millis et al., 1977,1978; Nicholson et al., 1978, 1981; Sicardy et al., 1981; Tomita, 1977). 


\section{RINGS DYNAMICS}

\section{Unconfined rings}

It has been shown (Brahic, 1975,1977, 1981 and Goldreich and Tremaine, 1978a,b) that, after a very fast flattening of the order of few tens of collisions per particle, a three-dimensional system of inelastically colliding particles reaches a quasi-equilibrium state in which the thickness of the newly formed disc is finite and in which collisions still occur. The combined effect of differential rotation and inelastic collisions makes the disc spread very slowly, particles moving both inwards and outwards carrying out some angular momentum. In the absence of external confining forces, the spreading time is of the order of the time it takes particles to random walk a distance equal to the ring width. The larger are the particles, the larger is the rate of this spreading (Brahic, 1977).

Poynting-Robertson drag (due to impacts with photons of light) and plasma drag (due to collisions with magnetospheric plasma) limit the lifetime of the small particles of a planetary ring (Burns et al 1981). But the rate of orbital decay caused by these processes is inversely proportional to particle size. Large particles are not affected by these drags.

If Uranian rings formed with the planet, the particles larger than a few millimeters would be eliminated by radial diffusion and the particles smaller than a few millimeters would have fallen onto the planet under the influence of Poynting-Robertson and plasma drags. Furthermore, differential shear and interparticle collisions should generate diffuse boundaries.

The presence of narrow rings with sharp edges around Uranus indicates that the rings are either young or confined. The absence of old diffuse rings seems to indicate that a confinement mechanism is present.

\section{Resonances}

A number of authors have considered resonances with known satellites. The figure 7 , where the major satellite resonances are represented, is not very conclusive. There is not a simple one-to- 

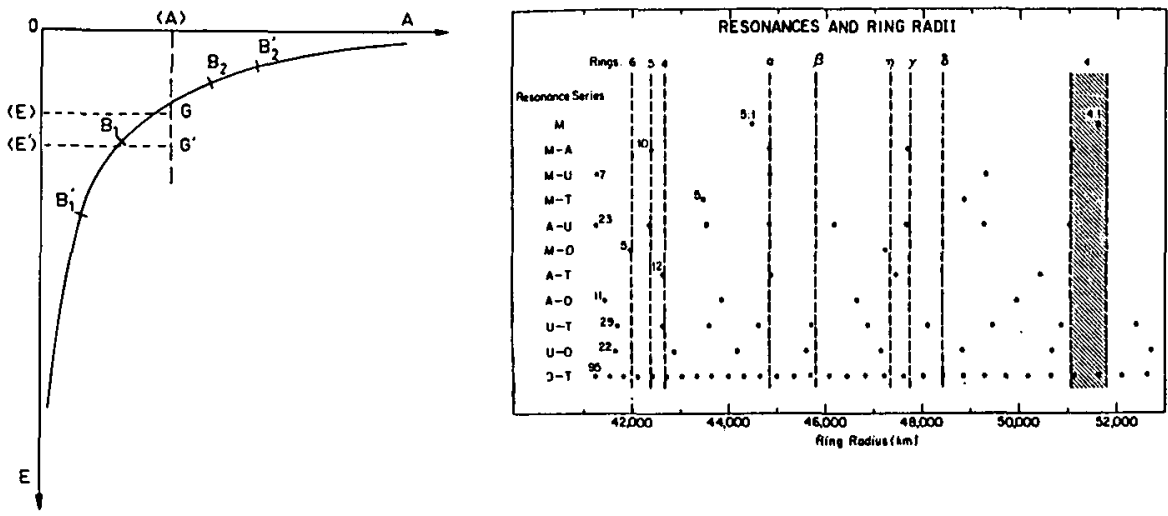

Figure 6. Angular momentum versus

Figure 7. Uranus's rings and energy for a disc in which each satellites. Resonances and ring particle is on a Keplerian orbit. radii. (After Elliot et al., The spreading disc is represented 1978). at the initial time by the arc $B_{1} B_{2}$ with barycentre $G$ and at $a$ latter time by the arc $B_{1}^{\prime} B_{2}^{\prime}$ with barycentre G'. This spreading is a direct consequence of the conservation of angular momentum, of the energy lost by collisions and of the concavity of the curve. (After Brahic, 1977).

one correspondence of resonant locations with ring structure. Furthermore the exchange of energy and angular momentum between the Uranian rings and the five known satellites of Uranus is not sufficient enough to confine the 9 narrow rings (Goldreich and Nicholson, 1977 and Aksness, 1977). Researchers now agree that resonances with moons outside the 9 rings are too weak to form most of the observed features directly.

\section{Horseshoe orbits}

Dermott, Gold et al. $(1977,1979,1980)$ proposed the interesting idea that each narrow ring contains a small satellite which maintains solid particles in stable, horseshoe orbits (as seen by an 
observer riding on the satellite) about its Lagrangian equilibrium points (Figure 8 ). This study is probably very appealing for a better understanding of the motion of the recently discovered coorbital satellites around Saturn. But inelastic collisions should destroy a ring of colliding particles (Aksness, 1977 and Goldreich and Nicholson, 1977) and it seems difficult to have a ring of confined particles at a location of a maximum of energy.

Cook and MacIntosh (198I) have proposed that satellites disintegrated inside Roche limit and are now dismembered into ring particles. The resulting condensed rings being a stable entity held together by its own gravitation.
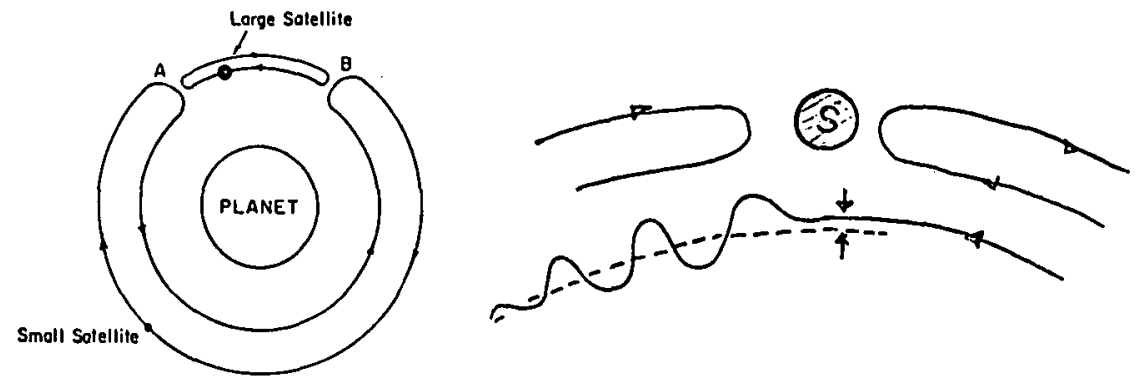

Figure 8. Left: Horseshoe orbit. (After Dermott, 1981).

Right: Ring particles paths in a frame rotating with the perturbing satellite. (From Goldreich and Tremaine, 1981).

\section{Guardian satellites}

A very interesting mechanism which is probably the right explanation has been quantitatively studied by Goldreich and Tremaine $(1979,1980,1981)$ and is now explored by Hénon (1981), Brahic (1981), Dermott (1981), Borderies (1981) and others.

Through gravity, a nearby satellite can alter a ring particle' s orbit, making it elliptical. The overall effect of this on a populous disc of particles is to increase in some places the density of particles and to decrease it in other places and finally to give rise to spiral density waves, like those in galaxies. If the perturbing satellite is exterior to the ring, this wave moves outwards, 
carrying negative energy and angular momentum. An isolated ring particle would return to its original position, but the rings are dense enough to make collisions between particles, and particles involved in such collisions finally move inward toward the planet. The outside satellite tends to take angular momentum from the ring and put to its orbit. Conversely, an inner satellite, moving faster than the particles of the ring, adds energy to nearby particles and transfers angular momentum to the ring from its orbit. This exchange of angular momentum have been first studied by Lin and Papaloizou (1979). A satellite near a planetary ring can push the ring away and simultaneously the satellite is repulsed. Small undetected satellites on each side of a ring could constrain its edges and prevent ring spreading, elliptical rings can also be generated by such a confining mechanism.

It is not intuitively obvious how a force of attraction can act to repel another body. This repulsion mechanism between a disc and a satellite depends on the presence of many ring particles that can often collide with each other. Changing a particle's angular momentum and thus its orbit depends on its having collisions or gravitational interactions (close encounters) with other particles. The physics involving many colliding particles sets the study of ring dynamics apart from classic celestial mechanics, in which few or no collisions are assumed, and fluid dynamics, in which much more frequent collisions are known to occur than in a planetary ring (the mean free path of an individual particle is much shorter in a fluid).

Satellites exert torques on the ring material at the locations of their low order resonance. For the simplest case of a circular ring and a circular satellite orbit, the strongest torques occur where the ratio of the satellite orbit period to the ring particle

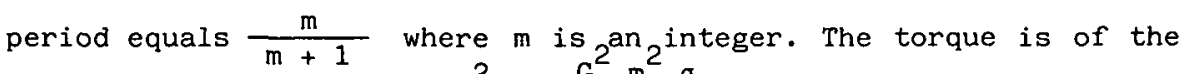
order of: $\quad \mathrm{T}_{\mathrm{m}} \sim \pm \mathrm{m}^{2} \frac{\mathrm{G}^{2} \mathrm{~m}_{\mathrm{s}}^{2} \sigma}{\Omega^{2} \mathrm{r}^{2}} \quad$ (Goldreich and Tremaine 1980,1981). G, $\Omega, \mathrm{m}_{\mathrm{s}}, \mathrm{r}$ and $\sigma$ are respectively the gravitational constant, the orbital frequency, the satellite mass, the radius and the surface mass density evaluated at the resonance location.

The spacing between neighbouring resonances from a nearby 
satellite is very small. The widths of individual resonances are greater than the frequency separations so that there are resonances that overlap. Thus it is useful to sum the discrete resonances torques and to define the total torque on a narrow ringlet of width $r$ :

$$
T \sim \pm \frac{G^{2} m_{s}^{2} \sigma r \Delta r}{\Omega^{2} x^{4}}
$$

where $x$ is the separation between the satellite and the ringlet ( $r \gg x \gg \Delta r$ ) (Goldreich and Tremaine, 1980).

The presence of dissipation is necessary. Although the expression for the torque does not reveal explicitely any dependence on dissipation, $T_{m}$ would vanish without dissipation. The exact nature of the dissipation does not affect the expression of the torque. Particles collisions are evidently the main source of dissipation.

This resonance torque does not act on isolated test particles. For example, there are not enough collisions in the asteroid belt and thus such a mechanism is not responsible for the formation of the Kirkwood gaps. The rate of transfer of angular momentum can also be calculated by a perturbative approach without reference to individual resonances. The gravitational force on a ring particle due to a satellite is only effective at close encounters. Since the tangential component of the relative velocity of the particle with respect to the satellite is reduced during encounter (Figure 8 ), angular momentum is exchanged with the net result that the ring experiences a torque (Lin and Papaloizou, 1979). During encounter, a particle initially moving on a circular orbit, acquires a radial velocity (Figure 8) and thereafter moves on a Keplerian ellipse. In a frame corotating with the perturbing satellite, all particles initially moving in circular orbits must follow similar paths after encounter. Thus each perturbing satellite generates a standing wave. In the inertial frame, each particle moves on an independent Keplerian ellipse, but the pericentres of these elliptical orbits and the phases of the particles on the orbits are such that the locus of the particles is a sinusoidal wave which moves through the ring with the angular velocity of the perturbing satellite. The damping of these waves, by collisions, results in a net exchange of angular momentum 
between the satellite and the ring particles.

This phenomenon is a sort of viscous dynamical friction studied in stellar dynamics. There are three main resonances for a Keplerian disc of infinite extent: one inner and one outer Lindblad resonance where the epicyclic motion of a particle in a circular orbit is strongly excited, since the perturbation frequency felt by the particle is equal to its epicyclic frequency and one corotation resonance where the angular momentum of one particle in a circular orbit undergoes large changes, since the particle feels a slowly varying azimuthal force (Figure 9 ).
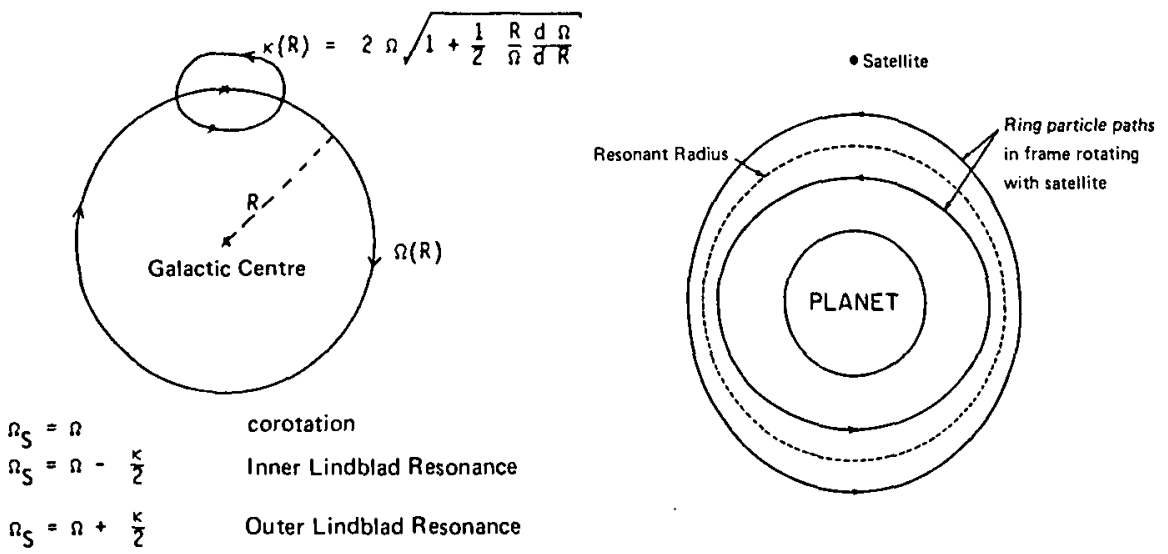

Figure 9. Left: Corotation and Lindblad resonances for galactic disc. Right: Particle paths in a frame rotating with the perturbing satellite in the vicinity of a resonance. The density of particles increases on the left and on the right and decreases in perpendicular directions. (After Dermott, 1981).

Planetary rings can support leading and trailing spiral density waves which are controlled by a combination of the Coriolis force and of the ring's self gravity. This phenomenon is similar to density waves in Messier 51. Close to a resonance, the long spiral waves have wavelengths several orders of magnitude greater than the interparticle spacing. These waves can exist only on the satellite side of the resonance and propagate toward and away from the resonance. The satellite excites the long trailing wave at the resonance and 
this wave carries away all of the angular momentum (positive or negative) which the resonance torque gives to the disc. The wave damps due to non linear and viscous effects close to the resonance. The particles on the satellite side of the resonance move toward the resonance. If the resonance torque is sufficiently large, a gap opens on the satellite side of the resonance. Goldreich and Tremaine (1978) have proposed this mechanism for producing the Cassini division in Saturn's rings due to the influence of Mimas which orbits well outside the rings.

Thus the structure of Uranian rings appears to require the existence of small, as yet undiscovered satellites which orbit within the ring system. We have no room here to describe in details the quantitative study of the interactions of the rings and these satellites, I give here only the main results of the brilliant work of Goldreich and Tremaine $(1979,1980,1981)$.

Torques not only transfer angular momentum but also energy, they can also change the eccentricity of the rings. Then torques can pump up the eccentricity of the rings, and the eccentricity of the satellites increase also, in other cases torques can damp the eccentricities of both the rings and the satellite. The $\varepsilon$ ring is probably associated with an eccentric satellite or two. If resonance torques are non linearly limited, both eccentricities of the ring and of the satellite will be non zero. Near corotation resonance, the eccentricity decreases. Near Lindblad resonance, the eccentricity increases (Goldreich and Tremaine, 1981).

Four different mechanisms have been explored by Goldreich and Tremaine (1979) to understand the precession of $E$ ring:

- The self gravity of the rings can maintain apse alignment if the mass of the satellite is at least of the order of $510^{18} \mathrm{~g}$. (that is much greater than that implied by the confinement theory) and if the mean surface density at quadrature is of the order of 25 g. $\mathrm{cm}^{-2}$.

- A nearly guardian satellite could force uniform precession in the ring. Goldreich and Tremaine have calculated the parameters of such a satellite.

- Smooth pressure gradients, due to interparticle collisions 
cannot produce uniform precession without an unreasonably large ring thickness.

- In a system of colliding particles, the possibility of a discontinuity analog to a shock which cannot be modelled by smooth pressure gradients shock-like phenomena could force uniform precession, but have not been analyzed.

Goldreich and Tremaine (1979) claim that apse alignment is maintained by the self-gravity of the ring. Their model predicts a strong variation in surface density profile as a function of true anomaly as a consequence of the non-uniform eccentricity gradient accross the ring. This prediction can be tested by future occultation observations.

In order to confine the observed narrow rings around Uranus, small satellite are sufficient. For example, kilometer-sized bodies are large and massive enough to confine the observed rings. They evidently cannot be observed from the Earth. If each Uranian ring is confined by at least two satellites, a minimum of 18 hypothetical satellites are necessary to confine all the rings. This has no observational support and it is probably why this theory of the guardian satellites has not been immediately accepted. In fact, the hypothetical satellites that confine the rings are not "external" satellites, but rather they are the largest "particles" in the rings.

Hénon (1981) has shown that, in a disc of particles of different sizes, a sufficiently massive particle will be able to push away the other disc particles, both towards the inside and the outside, and thus to clear completely an annular zone. As a consequence the particle population divides into:

a) the particles with a radius $r$ larger than a critical radius (which is $2.6 \mathrm{~km}$ in the case of Saturn's rings, each of which describes an isolated orbit and has a clear gap;

b) a "continuous" distribution of particles with a radius $r$ smaller than the critical radius and actually separated into a number of individual rings or ringlets by the massive particles. Brahic $(1979,1981)$ is doing a numerical simulation of a system of colliding particles orbiting around a central mass and perturbed by a satellite. Contrary to other approaches like Monte-Carlo 


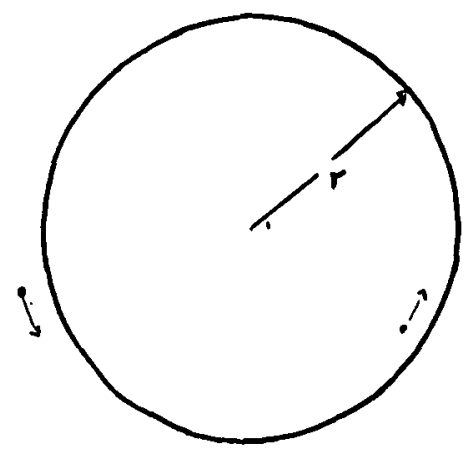

Figure 10. Two guardian satellites respectively inward and outward a ring at a distance $r$ from the center of the planet.
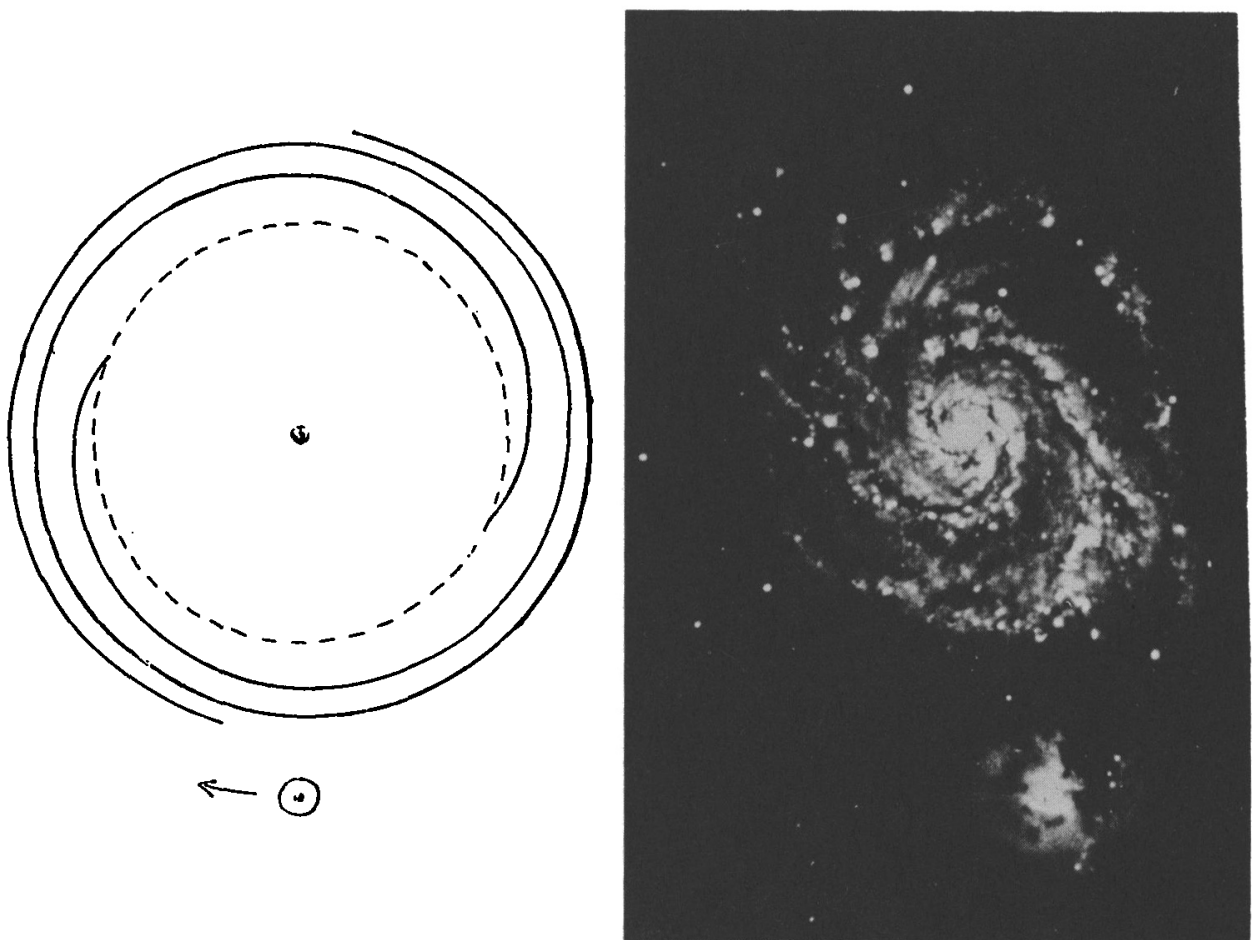

Figure 11. Left: a perturbing satellite generates a density wave in a ring of colliding particles.

Right: the galaxy Messier 51 and its companion which is probably responsible for the grand design of waves. 
methods, this model has the advantage to conserve very accurately the total angular momentum. The rate of transfer of angular momentum is calculated as a function of the mass of the satellite and its distance to the ring of colliding particles. The role of the most important resonances is particularly studied and the orbit of individual particles is calculated. There is no room here to enter into the details of the results which are presented elsewhere (Brahic, 1981).

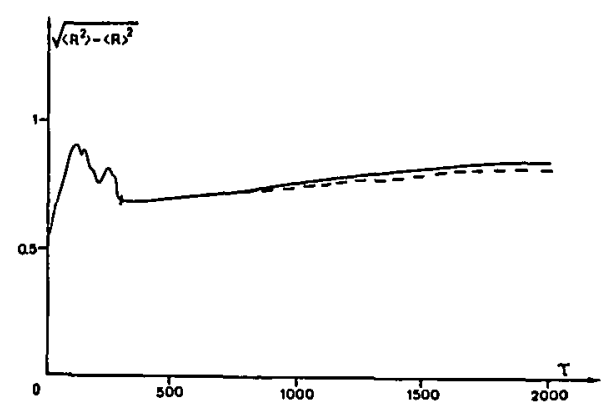

Figure 12. Spreading of the disc of colliding particles as a function of time. The full line represents the evolution of the disc without a perturbing satellite and the dashed line the evolution with a perturbing satellite (the disc is confined).

\section{OTHER PLANETARY RINGS}

The existence of ring systems around a planet is a natural consequence of collisions between ring particles and of the Roche limit. A cloud of particles moving around a central mass is rapidly flattened to a thin disc in a mean plane called the Laplacian plane which is identical to the equatorial plane of the planet in its vicinity. But unlike the planet's accumulation, large objects cannot grow within planetary rings because almost always these rings lie within what we call the Roche limit, inside which the self-gravity of an object is smaller than tidal stresses. Roche (1847) derived that, within a certain radial distance $d$ to the centre of a planet of radius $r$ and density $u_{p}$, a liquid satellite of density $u_{s}$ in synchronous rotation around the planet. This distance is $2.446\left(u_{p} / u_{s}\right)^{1 / 3} R$. The Roche limit, valid for an orbiting liquid body, has been used, often incorrectly, in the analysis of the break-up and accretion of solid bodies (see Smoluchowski, 1979 for a discussion). Because of their material strength, solid satellites can approach closer than the 
Roche limit, but if they exceed a certain size, they are broken apart by tidal forces (Aggarval and Oberbeck, 1974). The resulting fragments will continually reimpact one another, so that the largest member is not larger than some tens of kilometers.

We know now three systems of planetary rings. A quick look at them can lead to the conclusion that they are not at all similar. Seen from the Earth, Saturn has bright rings separated by narrow gaps, like a negative print of the dark narrow Uranian rings separated by large gaps while the main Jovian ring, closer to its central planet, is intermediate in size and has a three-dimensional halo. The Neptunian ring is suspected but not yet discovered (and even some authors claim that, as a consequence of the retrograde movement of Triton, it does not exist). These three known systems have been seen as perhaps more individualistic than the planet they surround. In fact, Voyager exploration has revealed that Jovian and Saturnian rings (Owen et al., 1979, Jewitt, 1981 and Science, 1981) have many common characteristics with Uranian rings:

- Resonances: Like in the case of Uranian rings, most radial ring structure of Jovian and Saturnian rings seems to be uncorrelated with the simple perturbations of known satellites: some strongly perturbed locations contain materials, other do not, some gaps are at resonant positions, most are not. Only a few prominent features of Saturn's rings appear to be associated with satellites'resonances. The best example is given by the Cassini division, which contains itself almost one hundred rings: Mimas clears a gap at the location of the 2:1 resonance and there is a relative absence of matter in the Cassini division, in comparison to adjacent parts of the $A$ and $B$ rings (Goldreich and Tremaine, 1978).

- Narrow rings: Close-up photographs by Voyager disclosed Saturn's rings to be more finely divided than anticipated. Rings lay within rings: upwards a thousand have been counted but, there are probably more at ascale narrower than voyager could resolve. The previously advanced explanation for the a priori perplexingly narrow rings of Uranus can also explain the narrowest rings of Saturn and may account for the grooved, "phonograph" appearance of the major rings' subdivisions (Figure 13) (Goldreich and Tremaine, 1979,1980 and 


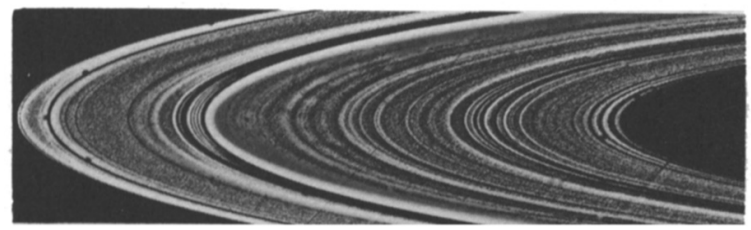

Figure 13. The rings of Saturn in a computer-processed image from Voyager 1. The outermost $F$ ring can be seen on the left with one of the two guardian satellites that "bind" its edges.

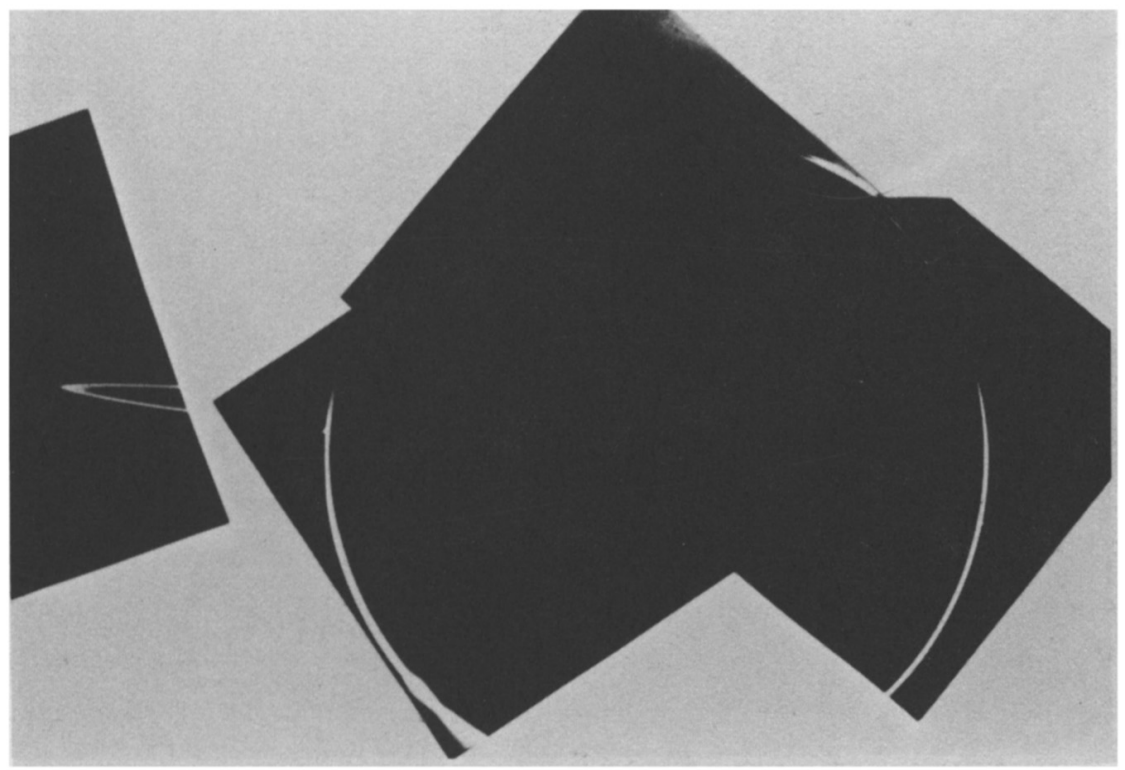

Figure 14. The ring of Jupiter seen by Voyager 2 from the planet's night side. The ring is unusually bright because of forward scattering from small particles within it. Similarly, the planet is outlined by sunlight scattered from a haze layer on the high atmosphere of Jupiter.

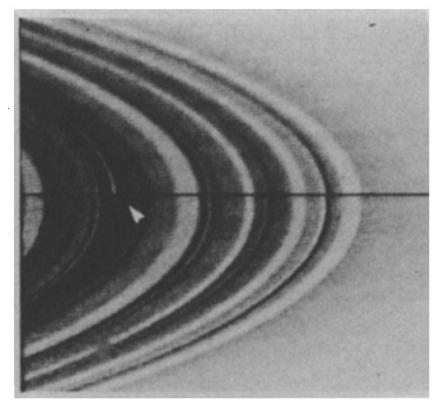

Figure 15. An elliptical ringlet (arrow) in the $C$ ring of Saturn. The figure was made in joining together two voyager 1 images of opposite ansae. 
Hénon, 1981).

The Jovian ring is optically thin and appears to contain little structure. Because of smear motion, features smaller than 700 kilometres cannot be resolved in the Voyager images (Jewitt and Danielson, 1981).

Herding by satellites or larger than average ring particles (if the two can be distinguished any longer) seems to be a widespread phenomenon and may explain most of Saturn's and Uranus's ring structure. Large objects within the disc itself not only perturb nearby material and probably explain the intricate structure of the rings, but they can also serve as sources and sinks of ring matter (Burns et al., 1981).

- Eccentric rings: Voyager's images of Saturnian rings show one eccentric ring in the $\mathrm{C}$ ring of Saturn, another in the Cassini division, and perhaps a third in the F ring itself.

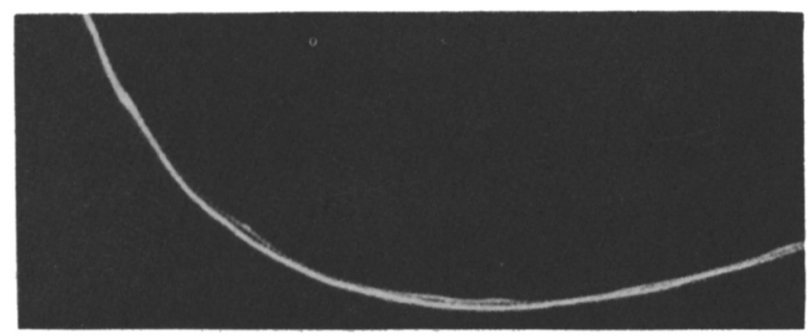

Figure 16. The F ring of Saturn. (N.A.S.A. document).

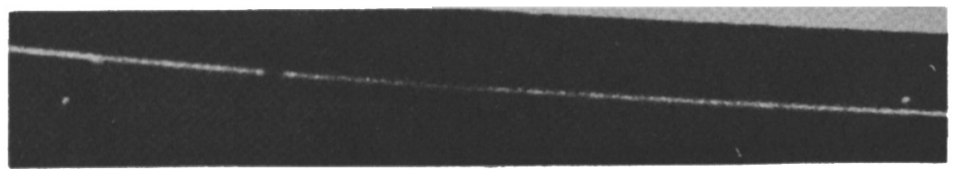

Figure 17. Segment of the $F$ ring showing the position of the neighbouring satellites $1980 \mathrm{~S} 26$ (left) and $1980 \mathrm{~S} 27$ (right). The outer edge of the $A$ ring is visible in the upper right. (N.A.S.A. document).

- Sharp edges: The discovery of small satellites just outside the Jovian ring lent credibility to theories which advocate the gravitational action of unseen satellites on the orbits of the rings' 
particles and this has been enhanced by the results of the Voyager 1 Saturn encounter. The outer A ring of Saturn and the main Jovian ring have both a sharp outer edge with a small satellite orbiting just at the outside of the ring, like a guardian satellite.

- Guardian satellites: The discovery of the narrow $F$ ring around Saturn and of two nearby satellites 1980 S 26 and 1980 S 27 respectively outward and inward (Figure 17) is a great success for the guardian satellite theory. There is a 3500 kilometres gap between this narrow $F$ ring (which voyager resolved into three rings 30 to 50 kilometres wide, two of which appear to be interwoven) and the outer A ring. Although the actual geometrical relationships of these F rings and the "braided" structure are still to be worked out, it seems that the Uranian rings are not so unruly. Gravitational perturbations of the F ring particles'orbits by 1980 S 26 and 1980 S 27 not only account for confinement of the ring particles but probably explain some of the structure of the F ring. Goldreich (1981) notes that the herding satellites, being vastly larger than those suggested for Uranus, should set up waves in the rings, which could explain at least some of their irregular shapes. Furthermore microscopic particles of the F ring, whose presence is evidenced by the ring's brightness when back-lighted on Voyager's images, are electrically charged and pushed about by Saturn's magnetic field. We do not know yet the distribution of particle sizes in Uranian rings. Dermott (1981) has studied the wave pattern formed in a ring by a nearby satellite.

It is clear now that the same fundamental mechanisms (interparticles'collisions, guardian satellites, resonances, electromagnetic forces on small particles,...) can explain much of the structure of the three known systems of rings. Nevertheless they are different and even inside Saturn the division into A, B and C rings is still meaningful and significant. The different distribution of sizes, the different nature of ring particles is not yet explained and is probably a result of different initial conditions and an illustration of how large is the number of solutions of Newton's equations. 


\section{ORIGIN OF THE SOLAR SYSTEM AND OTHER FLAT DISCS}

Aside from satisfying our curiosity, studies of rings can shed some light on how the diffuse ball of gas and dust, from which the solar system formed, could have been subdivided and concentrated to produce planets, moons, asteroids, comets, and rings systems themselves.

We do not know if planetary rings are the result of the tidal break-up of a large satellite or of the halted accretion of primitive material. But, whatever are initial conditions, the dynamical processesoccurring presently in planetary rings may provide an appropriate analog for events in the early solar system. Detailed structures visible today could be a fossil record of an intermediate stage in the accretion of orbiting bodies. Goldreich and Tremaine (1980) have presented an application of their study of disc-satellite interactions to the influence of the proto-Jupiter on the protoplanetary disc. In particular, the transfer of angular momentum in the protoplanetary disc and the role of resonances on the variations of orbits eccentricities (and thus on the relative velocities of the colliding planetoids) have to be explored in more detail. Models of planetary rings can be a good guide.

Techniques used for galactic dynamics have been particularly fruitful for the understanding of planetary rings. Conversely, a detailed study of rings structure can lead to a better understanding of spiral galaxies and accretion discs.

\section{FUTURE WORK}

Although the rings of Uranus may not be quite as mysterious as reported at first, much work remains for theoreticians who study ring dynamics. Goldreich and Tremaine made a particularly important step, but their study is only linear, we need now a non linear approach. It is difficult to predict future advances, but we can note that, so far, no calculations have been done in the case of large eccentricities. The transfer of angular momentum at the edge of a thick disc and the associated time scales have to be studied. The interaction of the magnetosphere of Uranus with small particles in 
Uranian rings, the nature of the particles, the origin of the rings and many other problems have still to be solved.

New rings, new satellites, new features (detection of broad and diffuse components of the ring system) and much more detailed structures can be found in Uranian rings. It is necessary to observe many more occultations at least to have a coverage as complete as possible of the ring system. Klemola et al. (1981) and Mink et al. (1981) give the dates of the next star's occultations by Uranus and Neptune visible from the Earth. At this conference, Elliot discusses tha data we can expect from next occultations, Voyager and the Space Telescope.

In order to have a better understanding of planetary rings and more generally of processes which have led to the formation of planets and satellites, it should be particularly important to observe individual "stones" in the ring at least up to a few meters. Only a sophisticated orbiter can perform this. Galileo (for Jupiter 's rings) and S.O.P.2 (for Saturn's rings) will be already particularly useful.

The amount of work is still so large that we can again thank Herschell for its discovery, even if he told, a little incautiously in 1794: "...I therefore venture to affirm that Uranus has no ring in the least resembling that, or rather those, of Saturn."

I would like to express gratitude and thank very warmly many of my colleagues for all the information they gave me and for all the discussions we had. I acknowledge in particular J. Burns, A. Cazenave, J. Elliot, P. Goldreich, R. Greenberg, M. Hénon, J. Lecacheux, P. Nicholson, T. Owen, B. Sicardy, S. Tremaine.

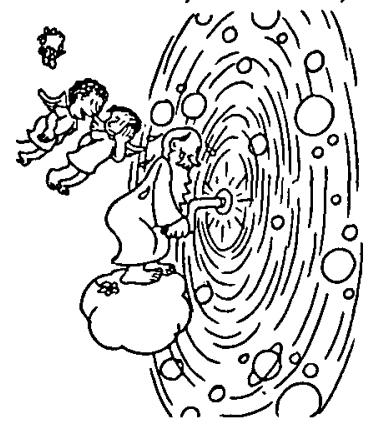

It is not so easy to start a planetary ring with a crank-handle! (After Jean Eiffel). 


\section{REFERENCES}

Aggarual,H.R., and Oberbeck,V.R. (1974). Roche limit of a solid body. Astrophys.J. 191, 577-588.

Aksness,K. (1977). Quantitative analysis of the Dermott-Gold theory for Uranus's rings. Nature 269, 783.

Bhattacharyya,J.C., and Bappu,M.K.V. (1977). Saturn-like ring system around Uranus. Nature 270, 503-506.

Borderies,N. (1981), preprint.

Brahic,A. (1975). A numerical study of a gravitating system of colliding particles: applications to the dynamics of Saturn's rings and to the formation of the solar system. Icarus 25, 452-458.

Brahic,A. (1977). Systems of colliding bodies in a gravitational field. I. Numerical simulation of the standard model. Astron: Astrophys; 54, 895-907.

Brahic,A. (1979). Influence of a satellite on the dynamical evolution of planetary rings. Bull. A.A.S. 11, 558.

Brahic,A., and Sicardy,B. (1981). Apparent thickness of Saturn's rings. Nature $289,447-450$.

Brahic,A. (1981). Planetary rings. In Formation of planetary systems (A. Brahic, Ed.)C.N.E.S. Paris.

Brahic, A. (1981). Preprint.

Brown, L.W. (1976). Astrophys. J. 207, L 209.

Chen,D.H., Yang,H.Y., Wu,Y.C., Kiang,S.Y., Huang,Y.W., Yeh,C.T., Chai,T.S., Hsieh,C.C., Cheng,C.S., and Chang,C. (1978). Photoelectric observation of the occultation of SAO 158687 by Uranian ring and the detection of Uranian ring signals from the light curve. Scientia Sinica XXI, 503-508.

Cook,A.F., and Mac Intosh,B.A. (1981). The rings of Uranus. Nature, submitted.

Dermott,S.F., and Gold,T. (1977). The rings of Uranus: theory. Nature 267, 590-593.

Dermott,S.F., Gold,T., and Sinclair,A.T. (1979). The rings of Uranus nature and origin. Astron. J. 84, 1225-1234.

Dermott,S.F., Murray,C.D., and Sinclair,A.T. (1980). The narrow rings of Jupiter, Saturn and Uranus. Nature 284, 309-313. 
Dermott,S.F., and Murray,C.D. (1980). Origin and eccentricity gradient and the apse alignment of the epsilon ring of Uranus. Icarus 43, 338-349.

Dermott,S.F. (1981a). The "braided" F ring of Saturn. Nature 290, 454-457.

Dermott,S.F. (1981b). The origin of planetary rings. Phil. Trans. R. In press.

Elliot,J.L., Dunham, E.W., and Mink,D.J. (1977a). The rings of Uranus Nature 267, 328-330.

Elliot,J.L., Dunham,E.W., and Millis,R.L. (1977b). Discovering the rings of Uranus. Sky Telesc. 53, 412-430.

Elliot,J.L., Dunham,E.W., Wasserman,L.H., Millis,T.L., and Churms,J. (1978). The radii of Uranian rings from their occultation of SAO 158687. Astron. J. 83, 980-992.

Elliot,J.L., Dunham,E., Mink,D.J., and Churms,J. (1980). The radius ellipticity of Uranus from its occultation of SAO 158687. Astrophys. J. 236,1026-1030.

Elliot,J.L., Frogel,J.A., Elias,J.H., Glass,I.S., French,R.G., Mink, D.J., and Liller,W. (1981). Orbits of nine Uranian rings. Astron. J. 86, 127-134.

Goldreich,P., and Nicholson,P.D. (1977). Revenge of tiny Miranda: Nature $269,783-785$.

Goldreich,P., and Tremaine,S. (1978). The velocity dispersion in Saturn's rings. Icarus $34,227-239$.

Goldreich,P., and Tremaine,S. (1978). The formation of Cassini division in Saturn's rings. Icarus 34, 240-253.

Goldreich, P , and Tremaine,S. (1979a). Towards a theory for the Uranian rings. Nature 277, 97-98.

Goldreich,P., and Tremaine,S. (1979b). Precession of the epsilon ring of Uranus. Astron. J. 243, 1638-1641.

Goldreich,P., and Tremaine,S. (1980). Disk-satellite interactions. Astrophys; J. 241, 425-441.

Goldreich,P., and Tremaine,S. (1981). The origin of the eccentricities of the rings of Uranus. Astrophys. J. 243, 1062-1075. Hénon, M. (1981) preprint. 
Hubbard,W.B., and Zellner,B.H. (1980). Results from the 10 March 1977 occultation by the Uranus system. Astron. J. 85, 16631669.

Ip,W.H. (1980). Physical studies of planetary rings. Space Sci. Rev. 26, 39-96.

Ip,W.H. (1980). New progress in the physical studies of planetary rings. Space Sci. Rev. 26, 97-109.

Jewitt, D.C. (1980). The rings of Jupiter. In the satellites of Jupiter (D. Morrison, Ed.). Univ. of Arizona Press, Tucson. Jewitt,D.C., and Danielson,G.E. (1980). The Jovian ring. J. Geophys. Res., in press.

Klemola,A.R., Mink,D.J., and Elliot,J.L. (1981). Predicted occultations by Uranus: 1981-1984. Astron. J. 86, 138-140.

Lin,D.N.C., and Papaloizou,J. (1979). Tidal torques on accretion discs in binary systems with extreme mass ratios. Mon. Not. Roy. Astron. Soc. 186, 799-812.

Mahra,H.S., and Gupta,S.K. (1977). Occultation of SAO 158687 by Uranian rings. I.A.U. Circ. n० 3061 .

Matthews,K., Nicholson,P.D., and Neugebauer,G. (1981). 2.2 microns maps of the rings of Uranus. preprint.

Millis,R.L., Wasserman,L.H., and Birch,P. (1977). Detection of the rings of Uranus. Nature 267, 330-331.

Millis,R.L., and Wasserman,L.H. (1978). The occultation of BD-150 3969 by the rings of Uranus. Astron. J. 83, 993-998.

Mink,D.J., Klemola,A.R., and Elliot,J.L. (1981). Predicted occultations by Neptune: 1981-1984. Astron. J. 86, 135-137.

Nicholson, P.D., Persson,S.E., Matthews,K., Goldreich,P., and Neugebauer,G. (1978). The rings of Uranus: results of the 1978 10 April occultation. Astron. J. 83, 1240-1248.

Nicholson,P.D., Matthews,K., and Goldreich,P. (1981). The Uranus occultation of 10 June 1979. I. The rings. Astron; J. 86, 596. Nicholson,P.D., Matthews, K., and Goldreich,P. (1981). Radial widths, optical depths and eccentricities of the Uranian rings,preprint Owen,T., Danielson,G.E., Cook,A.F., Hansen,C., Hall,V.L., and Duxburry,T.C. (1979): Jupiter's ring. Nature 281, 442-446. Science, vol.212, n 4491, 10 April 1981. 
Sicardy,B., Boucher,P., and Perrier, C. (1981). 15 August 1980 occultation by Uranus. preprint.

Smoluchowski,R. (1979). Planetary rings. Comments Astrophys. 8 , 69-78 Tomita,K. (1977). Observation of occultation of the SAO 158687 star by Uranus at Dodaira station. Tokyo Astron. Bull. 250. 